\title{
Fluidized Hot Melt Granulation: Influence of Process and Formulation Parameters
}

\author{
I. MAšı́́, A. Stojković, J. PAROJČıć, Z. ĐuRIĆ \\ Department of Pharmaceutical Technology, Faculty of Pharmacy, University of Belgrade, Serbia \\ E-mail: ivana.masic@pharmacy.bg.ac.rs (I. Mašić)
}

Sci Pharm. 2010; 78: 638

doi:10.3797/scipharm.cespt.8.PMS11

\begin{abstract}
Introduction: Hot-melt granulation techniques have received increasing attention due to the significant advantages compared to the conventional granulation methods. Fluidized hot melt granulation (FHMG) is recognized as promising technique, but there is still lack of information related to this topic [1]. The aim of the present study was to investigate the influence of process and formulation parameters on granule characteristics and paracetamol dissolution from tablets. Experimental methods: Granulation process was performed in Mycrolab fluid bed processor, OYSTAR Hüttlin. Precirol ATO 5 (Gattefossé, France) and PEG 2000 (Fluka AG, Switzerland) were used as meltable binders that were initially present as discrete particles within fluidized bed. The effects of binder content, particle size of the binder, granulation time, and the inlet air speed on the granule flow properties (Carr index) and drug dissolution parameters $\left(T_{40}, T_{80}\right)$ were investigated using factorial design.

Results and discussion: Formulations with Precirol ATO 5 showed considerably slower drug release due to the forming of the lipid matrix. The binder content had significant influence on granule properties, as well as on drug release rate in the case of both formulations with Precirol ATO 5 and formulations with PEG 2000. Concerning the paracetamol dissolution from tablets prepared with Precirol ATO 5, particle size and granulation were also found to be significant factors. Furthermore, two-factor interaction between the binder content and the particle size was identified.

Conclusion: FHMG is simple and rapid granulation technique that enables a formulator to manipulate drug release rate depending on the nature and concentration of the meltable binder.
\end{abstract}

[1] Andrews GP. Advances in solid dosage form manufacturing technology. Philos Trans R Soc Lond A. 2007; 365: 2935-2949. doi:10.1098/rsta.2007.0014 